\title{
APPELL POLYNOMIALS WHOSE GENERATING FUNCTION IS MEROMORPHIC ON ITS CIRCLE OF CONVERGENCE
}

\author{
BY J. D. BUCKHOLTZ
}

Communicated by W. T. Martin, October 19, 1972

Let $\Phi(z)=\sum_{0}^{\infty} \beta_{j} z^{j}$ have radius of convergence $r(0<r<\infty)$ and no singularities other than poles on the circle $|z|=r$. The Appell polynomials generated by $\Phi$ are given by

$$
\pi_{k}(z)=\sum_{j=0}^{k} \beta_{k-j} z^{j} / j !, \quad k=0,1,2, \cdots
$$

An entire function $g$ is said to possess a $\left\{\pi_{k}\right\}$ expansion if there is a complex sequence $\left\{h_{k}\right\}_{0}^{\infty}$ such that

$$
\sum_{k=0}^{\infty} h_{k} \pi_{k}(z)
$$

converges uniformly on compact sets to $g(z)$. In this note we show that the family of functions which have $\left\{\pi_{k}\right\}$ expansions is completely determined by the poles of $\Phi$ on $|z|=r$ together with the zeros of $\Phi$ in the closed disk $|z| \leqq r$.

Set $\Phi(z)=T(z) \phi_{1}(z) / P(z)$, where $\phi_{1}$ is analytic and zero-free in $|z| \leqq r$ and $T$ and $P$ are polynomials whose zeros correspond respectively to the zeros of $\Phi$ in $|z| \leqq r$ and the poles of $\Phi$ on $|z|=r$. Let

$$
P(z)=\prod_{q=1}^{\lambda}\left(1-\alpha_{q} z\right)^{m(q)}
$$

where $m(q)$ denotes the multiplicity of the pole $\alpha_{q}^{-1}$ of $\Phi$, and let $m=\max m(q), 1 \leqq q \leqq \lambda$. It is relatively easy to characterize those complex sequences $\left\{h_{k}\right\}_{0}^{\infty}$ for which (1) converges. The following result was proved in [2], and can also be obtained as a special case of a theorem of W. T. Martin [3].

THEOREM A. If $\left\{h_{k}\right\}_{0}^{\infty}$ is a complex sequence, then the following are equivalent:

(i) each of the series

$$
\sum_{k=0}^{\infty}\left(\begin{array}{c}
k+m(q)-1 \\
m(q)-1
\end{array}\right) h_{k} \alpha_{q}^{k}, \quad 1 \leqq q \leqq \lambda,
$$

converges;

AMS (MOS) subject classifications (1970). Primary 30A62; Secondary 30A16.

Key words and phrases. Appell polynomials, polynomial expansions.

Copyright (C) American Mathematical Society 1973 
(ii) the series (1) converges for all $z$ in some infinite bounded set;

(iii) the series (1) converges for all $z$, the convergence being uniform on every compact set.

The problem of determining which entire functions $g$ possess $\left\{\pi_{k}\right\}$ expansions is considerably more intricate, and the solution of this problem is our main result. Let

$$
Q(z)=\prod_{q=1}^{\lambda}\left\{1-\alpha_{q} z\right\}^{\min \{m(q), m-1\}},
$$

let $D$ denote the derivative operator, and let $\mathscr{F}$ denote the space of entire functions $f$ such that

$$
\lim _{n \rightarrow \infty} r^{-n}\left(D^{n} f\right)(0)=0 \quad \text { and } \quad \lim _{n \rightarrow \infty} n^{m-1} r^{-n}\left(Q(D) D^{n} f\right)(0)=0 .
$$

If $m=1$ ( $\Phi$ has only simple poles on $|z|=r$ ), the second condition reduces to the first, and $\mathscr{F}$ is the collection of all $f$ such that $f^{(n)}(0)=o\left(r^{n}\right), n \rightarrow \infty$. In general, $f^{(n)}(0)=o\left(r^{n}\right)$ is a necessary condition that $f$ belong to $\mathscr{F}$, and the condition

$$
f^{(n)}(0)=o\left(r^{n} / n^{m-1}\right), \quad n \rightarrow \infty,
$$

is sufficient. For each $k \geqq 0$, let $L_{k}$ denote the linear functional given by

$$
L_{k}(f)=\sum_{j=k}^{\infty} a_{j-k} f^{(j)}(0),
$$

where $\sum a_{j} z^{j}$ is the power series for $T(z) / \Phi(z)$. It was shown in [2] that, if $\Phi$ is zero-free in $|z| \leqq r$, then $g$ possesses a $\left\{\pi_{k}\right\}$ expansion if and only if $g$ belongs to $\mathscr{F}$. The expansion in this case is unique, the coefficient sequence $\left\{h_{k}\right\}_{0}^{\infty}$ being given by $\left\{L_{k}(g)\right\}_{0}^{\infty}$ (provided one takes $T(z) \equiv 1$ ). There is an easy and beautiful extension of this result to the general case.

THEOREM B. A necessary and sufficient condition that an entire function $g$ possess $a\left\{\pi_{k}\right\}$ expansion is that the differential equation $T(D) f=g$ have a solution $f$ which belongs to $\mathscr{F}$. If

$$
g(z)=\sum_{k=0}^{\infty} h_{k} \pi_{k}(z)
$$

for all $z$, then there is an $f \in \mathscr{F}$ such that $T(D) f=g$ and $h_{k}=L_{k}(f)$, $k=0,1,2 \ldots$ Conversely, if $f \in \mathscr{F}$ and $g=T(D) f$, then

$$
g(z)=\sum_{k=0}^{\infty} L_{k}(f) \pi_{k}(z)
$$

for all $z$, the convergence being uniform on every compact set. 
Proof. Let $\left\{p_{k}\right\}_{0}^{\infty}$ denote the Appell polynomial sequence generated by $\phi(z)=\Phi(z) / T(z)$; since $\phi$ is zero-free in $|z| \leqq r$, all the results obtained in [2] apply. Suppose that

$$
g(z)=\sum_{k=0}^{\infty} h_{k} \pi_{k}(z)
$$

for all $z$ and set

$$
f(z)=\sum_{k=0}^{\infty} h_{k} p_{k}(z) .
$$

It follows from Theorem A that the convergence of (3) is equivalent to that of (4), and is uniform on compact sets in both cases. Verify that $\pi_{k}=T(D) p_{k}$ and apply the operator $T(D)$ to both sides of (4). This yields

$$
(T(D) f)(z)=\sum_{k=0}^{\infty} h_{k} \pi_{k}(z)=g(z) .
$$

From (4) and the remark preceding Theorem B, it follows that $f \in \mathscr{F}$ and that $h_{k}=L_{k}(f), k=0,1,2, \cdots$.

Suppose now that $f \in \mathscr{F}$ and $g=T(D) f$. From the remark preceding Theorem B, we have

$$
f(z)=\sum_{k=0}^{\infty} L_{k}(f) p_{k}(z) .
$$

Applying $T(D)$ to both sides of (5), we obtain (2), and this completes the proof.

Unless $\Phi$ is zero-free in $|z|<r$, the $\left\{\pi_{k}\right\}$ expansions are not unique. Let $\mathscr{H}$ denote the space of all sequences $\left\{h_{k}\right\}_{0}^{\infty}$ such that

$$
\sum_{k=0}^{\infty} h_{k} \pi_{k}(z)=0
$$

for all $z$ (equivalently, for all $z$ in some infinite bounded set). Set $T(z)=T_{0}(z) T_{1}(z)$, where $T_{0}$ is a polynomial with no zero outside the disk $|z|<r$ and $T_{1}$ is a polynomial with no zero off the circle $|z|=r$. Let $\mathscr{H}_{0}$ denote the space of all sequences $\left\{h_{k}\right\}_{0}^{\infty}$ such that

$$
u(z)=\sum_{k=0}^{\infty} h_{k} z^{k} / k !
$$

satisfies the differential equation $T_{0}(D) u=0$. It is known (and easy to prove) $\left[\mathbf{1}\right.$, p. 25] that $\mathscr{H}_{0} \subseteq \mathscr{H}$. We shall prove that $\mathscr{H}=\mathscr{H}_{0}$ by showing that the dimension of $\mathscr{H}$ does not exceed the degree of $T_{0}$, which is the dimension of $\mathscr{H}_{0}$. This approach is necessary since our technique leads to a somewhat different characterization of $\mathscr{H}$. 
TheOREM C. $\mathscr{H}=\mathscr{H}_{0}$.

ProOF. Suppose $\left\{h_{k}\right\}_{0}^{\infty}$ belongs to $\mathscr{H}$. It follows from the argument used to prove Theorem B that the function $f(z)=\sum_{k=0}^{\infty} h_{k} p_{k}(z)$ belongs to $\mathscr{F}$ and satisfies $T(D) f=0$. Set $F=T_{0}(D) f$. Then

$$
0=T(D) f=T_{1}(D)\left\{T_{0}(D) f\right\}=T_{1}(D) F .
$$

Since $f \in \mathscr{F}$, it follows that $F \in \mathscr{F}$; therefore $F$ satisfies

$$
F^{(n)}(0)=o\left(r^{n}\right), \quad n \rightarrow \infty .
$$

The solutions of $T_{1}(D) F=0$ are well known, and the only one which satisfies (6) is $F \equiv 0$. Therefore $T_{0}(D) f=0$. The dimension of the solution space of $T_{0}(D) f=0$ is equal to the degree of $T_{0}$, and to complete the proof, we need only show that the linear mapping which takes the sequence $\left\{h_{k}\right\}_{0}^{\infty}$ in $\mathscr{H}$ onto the function $\sum h_{k} p_{k}(z)$ is $1-1$. This is equivalent to showing that $\sum h_{k} p_{k}(z)=0$ for all $z$ implies $h_{k}=0, k=0,1,2, \cdots$. This was established in [2]; therefore the proof is complete.

\section{REFERENCES}

1. R. P. Boas, Jr. and R. C. Buck, Polynomial expansions of analytic functions, Ergebnisse der Mathematik und ihrer Grenzgebiete, N.F., Band 19, Academic Press, New York; Springer-Verlag, Berlin, 1964. MR 29 \# 218.

2. J. D. Buckholtz, Appell polynomial expansions and biorthogonal expansions in Banach spaces, Trans. Amer. Math. Soc. (to appear).

3. W. T. Martin, On expansions in terms of a certain general class of functions, Amer. J. Math. 58 (1936), 407-420.

4. G. A. Read, Expansions in series of polynomials, J. London Math. Soc. 43 (1968), 655-657. MR 37 \# 4273.

Department of Mathematics, University OF Kentucky, LeXington, KentuCky 40506 